\title{
From drug-delivery device to disease management tool: a study of preferences for enhanced features in next-generation self-injection devices
}

This article was published in the following Dove Press journal:

Patient Preference and Adherence

\author{
Marco Boeri ${ }^{1}$ \\ Boglarka Szegvari ${ }^{2}$ \\ Brett Hauber ${ }^{3}$ \\ Brennan Mange ${ }^{3}$ \\ Irina Mountian ${ }^{2}$ \\ Michael Schiff ${ }^{4}$ \\ Nikolaos Maniadakis ${ }^{5}$ \\ 'RTI Health Solutions, Belfast, UK; ${ }^{2}$ UCB \\ Pharma, Brussels, Belgium; ${ }^{3}$ RTI Health \\ Solutions, Research Triangle Park, NC, \\ USA; ${ }^{4}$ Rheumatology Division, University \\ of Colorado School of Medicine, Denver, \\ CO, USA; ${ }^{5}$ Health Services Management, \\ National School of Public Health, Athens, \\ Greece
}

Purpose: To quantify rheumatology patient preferences and willingness to pay (WTP) for features differentiating enhanced from standard self-injection devices and to investigate differences among subgroups.

Patients and methods: Patients with rheumatoid arthritis (RA), psoriatic arthritis (PsA), and axial spondyloarthritis (axSpA) were recruited in the UK. A discrete-choice experiment was used to elicit preferences; respondents were presented with 10 choices between 3 different devices: a free standard disposable device, and 2 hypothetical reusable devices characterized by presence/absence of skin sensor, injection speed control, on-screen instructions, injection reminders, electronic log, and large grip. Every hypothetical device included a cost component to assess WTP for each enhanced feature. A random-parameters logit model was used to estimate preference weights and WTP.

Results: Data were collected from 323 respondents by electronic survey (15/11/2017-15/02/ 2018; RA: 108; PsA: 103; axSpA: 112). On average, the skin sensor was the most preferred feature $(£ 30)$, followed by injection speed control, injection reminders, electronic log $(\sim £ 20$ each), on-screen instructions ( $£ 12)$, and a device with a small, rather than large grip $(\sim £ 7)$. Similar preferences for attributes were observed across condition subgroups except for grip size: axSpA patients preferred small grip ( $£ 27)$; PsA patients preferred large grip $(\sim £ 19)$. Overall, respondents preferred reusable devices with all enhanced features (WTP value: £85) over the standard device. RA patients exhibited a higher WTP (£145) than PsA (£102) or axSpA (£62) for the same enhanced device.

Conclusion: Patients positively valued reusable self-injection devices with enhanced features, which may improve patient experience, potentially improving treatment adherence, clinical, and economic outcomes.

Keywords: rheumatology, subcutaneous injection, self-administration, patient preference, discrete-choice experiment

\section{Introduction}

Anti-tumor necrosis factors (anti-TNFs) are biologic disease-modifying antirheumatic drugs (bDMARDs) effective in treating multiple chronic inflammatory diseases, including rheumatoid arthritis (RA), axial spondyloarthritis (axSpA), and psoriatic arthritis (PsA). ${ }^{1,2}$ Anti-TNFs can be self-administered by subcutaneous injection. This method of administration can increase patient independence, improve patient satisfaction, and allow patients to gain better control of their treatment schedule. ${ }^{3-5}$

Although self-injection may improve patient treatment experience, ${ }^{5}$ there are several barriers to successful self-injection. Psychological and social barriers, such 
as injection anxiety, needle phobia, and lack of confidence, are reported among patients who self-inject. ${ }^{5}$ Physical barriers can also impede safe self-injection, such as hand dexterity problems and pain, which are commonly found in RA patients. ${ }^{5-7}$ Together, these barriers can lead to poor treatment adherence and, consequently, poor clinical and economic outcomes. ${ }^{2,4}$

A number of treatment delivery factors have been shown to impact patient medication adherence; optimizing device design to fulfill any unmet needs and meet patient expectations may enhance patient experience, improve disease management, and reduce treatment nonadherence. ${ }^{1}$ As a result, research into patient-reported preferences and outcomes has directed and advanced the development of self-injection devices. ${ }^{3}$ Evidence from focus groups suggests patient views on what constitutes an "ideal device" can vary, with patients valuing alternative features differently. ${ }^{8}$ Differences in preferences may be driven by a number of factors, for example, the impact of disease on joint mobility, patient lifestyle, chronic disease longevity and management, and fears and beliefs around disease. 9,10 Discrete-choice experiments (DCE) are routinely used to elicit individuals' preferences and can be used when comparing health care technologies. ${ }^{11,12}$ For example, a DCE study examining the preferences of RA patients highlighted differences in administration route preferences, demonstrating that subcutaneous injection was preferred when considering convenience. ${ }^{13}$

Reusable electromechanical self-injection devices (e-Devices) with a dose dispenser cartridge are now approved for delivery of anti-TNF treatments in Europe. ${ }^{14,15}$ Patient feedback from human factor studies was used in the development of e-Device designs to ensure the final design addressed patient needs more effectively than existing options. ${ }^{1}$ The e-Device incorporates a number of enhanced features including injection speed control to reduce patient anxiety and increase injection comfort, an injection $\log$ to track medication compliance, a "next injection" notification feature, a skin sensor to prevent injection misfire and drug wastage, step-by-step on-screen instructions and training cartridge to help increase patient confidence, and an ergonomic grip to enhance handling and control. ${ }^{14}$

The primary objective of this study was to use a DCE to elicit patients' preferences for a set of enhanced features that differentiate e-Devices from other currently marketed pre-filled pens. Other objectives included calculating patients' willingness to pay (WTP) for enhanced features, to quantify patients' relative preference for different hypothetical device profiles and features. Additionally, this study aimed to explore whether disease condition, disease severity, and self-injection experience were associated with systematic differences in preferences.

\section{Materials and methods}

\section{Patient eligibility and data collection}

Physicians in the UK were contracted by the healthcare research company, AplusA (London, UK), to invite patients with RA, PsA, and $\operatorname{axSpA}$ to participate in the study. Physicians (both GPs and specialists) who had previously agreed to refer patients for surveys were contacted by email or by phone and email. All patients were aged $\geq 18$ years, had a moderate or severe diagnosis of the conditions based on the physician's clinical judgment, were residents of the UK, and could read and understand English. Patients' physicians provided confirmation of disease condition and severity.

Once interest in study participation was received from the patient, a link to the study survey was sent via email. Initial questions were used to assess the patient's eligibility to participate. Eligible patients provided informed, electronic consent before being asked to complete the study survey. All patients were paid nominal remuneration to compensate them for their time to complete the study surveys. Patient demographic data including age, level of education, employment status, and household income were collected. Additionally, data about each patient's disease history were recorded, including time since diagnosis, disease severity and previous treatment and self-injection experience.

\section{Discrete-choice experiment}

Following recommended research practices, ${ }^{16}$ a DCE survey was developed to elicit preferences and WTP for a set of enhanced self-injection device features (skin sensor functionality, injection speed control, step-by-step onscreen instructions, injection date reminder, electronic $\log$ ) and having a large, instead of a small grip, in patients with RA, axSpA, and PsA. To select these enhanced device features to be assessed in the DCE, the study team reviewed focus group reports from the study sponsor, as well as the available literature. ${ }^{17,18}$ The study team then selected the device features that appeared to be most important to patients during the focus groups and which differentiated devices that may be available in the near future. Each DCE choice question included three device 
profiles: a standard disposable device with no enhanced features and a small grip, and two hypothetical reusable device profiles (reusable device A and reusable device B), characterized by different combinations of enhanced features. To elicit patients' WTP, the DCE included a cost attribute as a one-off payment for the device. The out-ofpocket cost of the standard disposable device was set to $£ 0$.

Before administering the DCE survey, 14 qualitative face-to-face pretest interviews and 1 telephone interview were conducted with a convenience sample of patients with RA, axSpA, or PsA from the UK. The interviews were used to ensure the survey was usable and understandable, and tested the levels of the "out-of-pocket" cost attribute of the survey. To ensure the "out-of-pocket" costs were reasonable, patients in the initial five pretest interviews completed the DCE assuming cost was constant between all devices and were then asked to suggest the cost they would be willing to pay for each feature.

The cost levels included in the hypothetical reusable device profiles ranged from $£ 15$ to $£ 250$. A scope test (or partial internal validity test) was performed to further confirm the cost was presented and interpreted correctly in the online survey, ie, that respondents evaluated a given difference in cost similarly, regardless of whether the cost change occurred within a narrow range of costs or within a wide range of costs. To perform the scope test, respondents were randomly assigned to 1 of 2 different split samples with different ranges of out-of-pocket cost: i) narrow range $(£ 0, £ 15, £ 30, £ 50, £ 90$, and $£ 150)$ and ii) wide range ( $£ 0, £ 15, £ 30, £ 50, £ 125$, and $£ 250) .{ }^{19}$

Although patients were asked to consider the out-ofpocket cost for a new device, UK patients do not currently pay for their devices. Therefore, to clarify that the purpose of the survey was to elicit the value, and not the price of the features, a short budget reminder, sometimes also referred to as "cheap talk" script, was included in the survey before the DCE questions (Supplementary Materials). ${ }^{19}$ The combinations of enhanced hypothetical reusable device features and out-of-pocket costs were determined using an experimental design developed using a D-efficient algorithm. ${ }^{20}$ This allowed for the estimation of a preference weight for each attribute level included in the study.

The survey was programmed and hosted online by AplusA between November 15, 2017 and February 15, 2018. Based on the number of device attributes, as well as the number of cost levels included, a total of 40 DCE questions was considered the optimal number to have sufficient observations to estimate the models. The experimental design therefore comprised 40 DCE questions, divided into 4 different blocks. Each respondent was assigned to 1 of the 4 blocks, each of which featured just 10 questions, to avoid individual respondents becoming fatigued. Within each block, the DCE questions were also randomized to mitigate ordering effects. An example DCE question is presented in Figure 1.

\section{Statistical analyses}

Descriptive analyses were conducted to summarize respondent demographics. Continuous variables were summarized using mean, standard deviation (SD), median, and range; categorical data were summarized using frequencies and percentages.

Responses to the DCE questions were analyzed using a random-parameters logit (RPL) error component model. ${ }^{21}$ "Out-of-pocket cost" was interacted with the natural log of the respondents' income to control for income effects and assumed to be a linear and a continuous function of the levels of "out-of-pocket cost" shown to respondents. Before DCE data modeling, a scope test on the out-ofpocket cost was performed on all collected data. This tested whether respondents paid attention to the absolute numeric values presented in the survey as out-of-pocket cost or interpreted the numeric levels presented qualitatively as high, medium, and low. ${ }^{19}$

All other attributes were categorical, and effects-coded as 1 when the attribute level "yes" was included in the profile and -1 when the attribute level "no" was included. An RPL error component model was estimated to capture preference heterogeneity for a hypothetical reusable device over a standard disposable device. ${ }^{22}$ The estimates from the RPL model were log-odds associated with each attribute level and were interpreted as relative preference weights. Each preference weight indicated the relative strength of preference for the attribute level, and a higher preference weight corresponded to a more preferred level of an attribute.

\section{Subgroup analysis}

Preference weights for the attributes included in the DCE were estimated for three prespecified subgroups of interest: condition (axSpA, PsA, RA); physician assessment of disease severity (severe, moderate); and self-injection experience (naïve, experienced). WTP was also calculated for each subgroup. Differences between subgroups by age and gender were also investigated. 


\begin{tabular}{|c|c|c|c|}
\hline Device feature & Reusable device A & Reusable device B & $\begin{array}{l}\text { Standard disposable } \\
\text { device }\end{array}$ \\
\hline Skin sensor 0 & No & No & No \\
\hline Control of the speed of injection & No & Yes & No \\
\hline $\begin{array}{l}\text { Step-by-step, on-screen } \\
\text { instructions }\end{array}$ & & No & No \\
\hline Injection date reminders 0 & & No & No \\
\hline Electronic log 0 & & & No \\
\hline \multirow[t]{2}{*}{ Grip size 0} & & & \\
\hline & Small grip & Large grip & Small grip \\
\hline Out-of-pocket cost 0 & $£ 125$ & $£ 30$ & $£ 0$ \\
\hline Which would you choose? & O & (2) & $\mathrm{O}$ \\
\hline
\end{tabular}

Figure I Example DCE question. The final experimental design included 40 sets of 3 devices split into 4 blocks of 10 hypothetical choice questions. Patients were randomly assigned to I block of 10 questions. Questions were randomly ordered to avoid ordering effects.

Interaction models were estimated for each subgroup to estimate differences in mean preferences between subgroups. A dummy variable indicating a specific subgroup was interacted with each attribute level in the study. The unobserved heterogeneity captured by the RPL was assumed to be constant across subgroups. The explanatory variables in the interaction model included both the full set of attribute levels and the full set of interactions. A Wald test was used to determine the joint statistical significance of differences between each subgroup. ${ }^{23}$

Analyses were conducted using NLOGIT 5.0 (Econometric Software, Plain View, NY, USA).

\section{Welfare analysis}

The inclusion of the out-of-pocket cost in the DCE forced tradeoffs between the benefit gained from the inclusion of a specific feature in the device and the loss caused by having to pay for it. The preference weight for the outof-pocket cost was interpreted as the incremental disutility caused by a one-off payment of one additional pound for the reusable device with a combination of enhanced features. The ratio between the utility gained when including the additional device feature and the disutility of cost (known as WTP) can be interpreted as the welfare gain a patient would obtain by including the additional feature in the device.

Since the experimental design included a reference self-injection device with a zero out-of-pocket cost and no enhanced features, it was possible to compute the value of specific combinations of enhanced features in a reusable self-injection device by the compensating variation (CV) (Supplementary Materials). ${ }^{24}$ The value associated with a reusable device with all the enhanced features and a large grip, compared to the standard disposable device with small grip was calculated.

\section{Ethical considerations}

The RTI International Institutional Review Board (IRB) determined that this study meets the criteria for IRB exemption under the Code of Federal Regulations (section 45CFR46.101(b) specifically) because the research involved the use of survey procedures, the information obtained was recorded in such a manner that human subjects could not be identified, directly or through identifiers linked to the subjects, and any disclosure of the human 
subjects' responses outside the research would not reasonably place the subjects at risk of criminal or civil liability or be damaging to the subjects' financial standing, employability, or reputation. In the qualitative pretest interviews, each interview participant signed and dated an informed consent form before the interview. All respondents to the DCE survey provided electronic informed consent and received nominal compensation for time spent participating. The study complied with the Declaration of Helsinki.

\section{Results}

\section{Patient characteristics}

Over 1,000 physicians were contacted and 37 agreed to recruit patients for the survey. Links to the online survey were sent via email to 415 patients, 323 $(77.8 \%)$ were eligible, provided informed consent, and completed the survey. The mean (SD) patient age was 48.6 years (13.7) and $200(61.9 \%)$ were female. Of the 323 patients, $151(46.7 \%)$ had no previous self-injection experience (Table 1). The demographics of patients stratified by condition are presented in Table 2. Of the 323 patients, 112 (34.7\%) were diagnosed with axSpA, 103 (31.9\%) with PsA and 108 $(33.4 \%)$ with RA. Within the disease subgroups, 53/103 (51.5\%) PsA patients, 57/108 (52.8\%) RA patients, and 51/112 (45.5\%) axSpA patients were classified by their physician as having moderate disease severity. 53/103 (51.5\%) PsA patients, 58/108 (53.7\%) RA patients, and 61/112 (54.5\%) axSpA patients had experience with self-injection (Table 3).

Table I Patient demographics, all patients $(\mathrm{N}=323)$

\begin{tabular}{|c|c|}
\hline & All patients, n (\%) \\
\hline Age, years, mean $(S D)^{a}$ & $48.6(13.7)$ \\
\hline \multicolumn{2}{|l|}{ Gender } \\
\hline Female & $200(6 \mid .9)$ \\
\hline Male & $122(37.8)$ \\
\hline Prefer not to say & $\mathrm{I}(0.3)$ \\
\hline \multicolumn{2}{|l|}{ Time since diagnosis } \\
\hline$<2$ years & $47(14.6)$ \\
\hline $2-<4$ years & $63(19.5)$ \\
\hline $4-<10$ years & $94(29.1)$ \\
\hline$\geq 10$ years & 117 (36.2) \\
\hline Unknown/unsure & $2(0.6)$ \\
\hline
\end{tabular}

(Continued)
Table I (Continued).

\begin{tabular}{|c|c|}
\hline & All patients, $\mathbf{n}(\%)$ \\
\hline \multicolumn{2}{|l|}{$\begin{array}{l}\text { Previous experience using a self-injection } \\
\text { device }\end{array}$} \\
\hline Yes & $172(53.3)$ \\
\hline No & I5I (46.7) \\
\hline \multicolumn{2}{|l|}{ Level of education } \\
\hline No formal education & $\mathrm{I}(0.3)$ \\
\hline Primary school education or less & $6(1.9)$ \\
\hline $\begin{array}{l}\text { Secondary school education up to general/ } \\
\text { ordinary/standard level }\end{array}$ & $72(22.3)$ \\
\hline $\begin{array}{l}\text { Secondary school education up to inter- } \\
\text { mediate/advanced/higher level }{ }^{c}\end{array}$ & $46(14.2)$ \\
\hline Professional or work-related qualifications ${ }^{d}$ & $79(24.5)$ \\
\hline Undergraduate university degree & 61 (18.9) \\
\hline Postgraduate university degree or equivalent & $45(13.9)$ \\
\hline Preferred not to answer & $13(4.0)$ \\
\hline \multicolumn{2}{|l|}{ Employment status } \\
\hline Employed, full-time & $112(34.7)$ \\
\hline Employed, part-time & $54(16.7)$ \\
\hline Self-employed & $21(6.5)$ \\
\hline Homemaker & II (3.4) \\
\hline Student & II (3.4) \\
\hline Retired & $56(17.3)$ \\
\hline Disabled/unable to work & $33(10.2)$ \\
\hline Unemployed but looking for work & $14(4.3)$ \\
\hline Unemployed and not looking for work & $2(0.6)$ \\
\hline Preferred not to answer & $9(2.8)$ \\
\hline \multicolumn{2}{|l|}{ Household income $e^{e}$} \\
\hline Less than $£ 25,000$ & $78(24.1)$ \\
\hline$£ 25,000$ to $£ 49,999$ & $90(27.9)$ \\
\hline$£ 50,000$ to $£ 74,999$ & $53(16.4)$ \\
\hline$£ 75,000$ to $€ 99,999$ & $24(7.4)$ \\
\hline$\notin 100,000$ to $f 149,999$ & $5(1.5)$ \\
\hline$f 150,000$ to $f 199,999$ & $\mathrm{I}(0.3)$ \\
\hline Unknown/unsure & $16(5.0)$ \\
\hline Preferred not to answer & $56(17.3)$ \\
\hline
\end{tabular}

Notes: ${ }^{a} n=322$, age unknown for I patient; ${ }^{b}$ CSEs, GCSEs, O-levels, Scottish Standard Grades, or equivalent; 'A/S levels, A-levels, Scottish Intermediates, Scottish Highers, International/Scottish Baccalaureate or equivalent; ${ }^{\mathrm{d} C e r t i f i c a t e}$ of Higher Education, Diploma of Higher Education, foundation degree; ${ }^{\mathrm{e} H}$ Household income (2016) before tax and other deductions.

Abbreviation: SD, standard deviation.

\section{DCE preference analysis}

The scope test, conducted by assigning half of the respondents to a narrow range ( $£ 0, £ 15, £ 30, £ 50, £ 90$, and $£ 150)$ and half to a wide range $(£ 0, £ 15, £ 30, £ 50, £ 125$, and $£ 250$ ) of out-of-pocket costs, suggested respondents paid attention to the absolute numeric values, rather than interpreting the numeric levels as qualitative categories such as 
Table 2 Respondent demographics by condition subgroup ( $N=323)$

\begin{tabular}{|c|c|c|c|c|}
\hline & $\begin{array}{l}\text { Respondents with } \\
\text { axSpA }\end{array}$ & $\begin{array}{l}\text { Respondents } \\
\text { with PsA }\end{array}$ & $\begin{array}{l}\text { Respondents } \\
\text { with RA }\end{array}$ & $\begin{array}{l}\text { All respon- } \\
\text { dents }\end{array}$ \\
\hline \multicolumn{5}{|l|}{ Age (years) } \\
\hline $\mathrm{n}$ & 112 & 103 & 107 & 322 \\
\hline Mean (SD) & $48.4(14.4)$ & $48.5(12.4)$ & $49.0(14.3)$ & $48.6(13.7)$ \\
\hline Median & 48.5 & 48.0 & 50.0 & 48.0 \\
\hline Min, $\max$ & 20,83 & 21,79 & 19,89 & 19,89 \\
\hline Missing & 0 & 0 & I & I \\
\hline \multicolumn{5}{|l|}{ Country of residence, $n$ (\%) } \\
\hline $\mathrm{N}$ & 112 & 103 & 108 & 323 \\
\hline England & $106(94.6)$ & $99(96.1)$ & $95(88.0)$ & $300(92.9)$ \\
\hline Scotland & $2(1.8)$ & $4(3.9)$ & $9(8.3)$ & $15(4.6)$ \\
\hline Wales & $4(3.6)$ & 0 & $2(1.9)$ & $6(1.9)$ \\
\hline Northern Ireland & 0 & 0 & $2(1.9)$ & $2(0.6)$ \\
\hline Other - EU country & 0 & 0 & 0 & 0 \\
\hline Other - non-EU country & 0 & 0 & 0 & 0 \\
\hline \multicolumn{5}{|l|}{ Condition diagnosis, ${ }^{\mathrm{a}} \mathrm{n}(\%)$} \\
\hline $\mathrm{n}$ & 112 & 103 & 108 & 323 \\
\hline Allergies & $12(10.7)$ & $10(9.7)$ & $8(7.4)$ & $30(9.3)$ \\
\hline axSpA & $112(100)$ & 0 & 0 & $112(34.7)$ \\
\hline Anxiety & $16(14.3)$ & $12(11.7)$ & $12(I I . I)$ & $40(12.4)$ \\
\hline Obesity & $9(8.0)$ & $9(8.7)$ & $6(5.6)$ & $24(7.4)$ \\
\hline Osteoarthritis & $10(8.9)$ & $10(9.7)$ & $16(14.8)$ & $36(11.1)$ \\
\hline COPD & 0 & $2(1.9)$ & I (0.9) & $3(0.9)$ \\
\hline Diabetes & $7(6.3)$ & $3(2.9)$ & $9(8.3)$ & $19(5.9)$ \\
\hline Depression & $22(19.6)$ & $16(15.5)$ & $18(16.7)$ & $56(17.3)$ \\
\hline Fibromyalgia & $7(6.3)$ & $9(8.7)$ & $9(8.3)$ & $25(7.7)$ \\
\hline High blood pressure/hypertension & $25(22.3)$ & $15(14.6)$ & $13(12.0)$ & $53(16.4)$ \\
\hline High cholesterol & $15(13.4)$ & II (I0.7) & $13(12.0)$ & $39(12.1)$ \\
\hline Insomnia & $3(2.7)$ & $6(5.8)$ & $3(2.8)$ & $12(3.7)$ \\
\hline PsA & $7(6.3)$ & $103(100)$ & 0 & $110(34.1)$ \\
\hline RA & $6(5.4)$ & $8(7.8)$ & $108(100)$ & $122(37.8)$ \\
\hline None of the above & 0 & 0 & 0 & 0 \\
\hline \multicolumn{5}{|l|}{ Gender, n (\%) } \\
\hline $\mathrm{n}$ & 112 & 103 & 108 & 323 \\
\hline Female & $50(44.6)$ & $64(62.1)$ & $86(79.6)$ & $200(6 \mid .9)$ \\
\hline Male & $62(55.4)$ & $38(36.9)$ & $22(20.4)$ & $122(37.8)$ \\
\hline Other & 0 & 0 & 0 & 0 \\
\hline Prefer not to answer & 0 & $\mathrm{I}(\mathrm{I} .0)$ & 0 & $\mathrm{I}(0.3)$ \\
\hline \multicolumn{5}{|l|}{ Marital status, n (\%) } \\
\hline $\mathrm{n}$ & 112 & 103 & 108 & 323 \\
\hline Single/never married & $34(30.4)$ & $17(16.5)$ & $21(19.4)$ & $72(22.3)$ \\
\hline Married/living as married/civil partnership & $56(50.0)$ & $70(68.0)$ & $73(67.6)$ & $199(61.6)$ \\
\hline Divorced or separated & $15(13.4)$ & $13(12.6)$ & $10(9.3)$ & $38(11.8)$ \\
\hline Widowed/surviving partner & $2(1.8)$ & $3(2.9)$ & $3(2.8)$ & $8(2.5)$ \\
\hline Other & $3(2.7)$ & 0 & 0 & $3(0.9)$ \\
\hline Prefer not to answer & $2(1.8)$ & 0 & I (0.9) & $3(0.9)$ \\
\hline
\end{tabular}

(Continued) 
Table 2 (Continued).

\begin{tabular}{|c|c|c|c|c|}
\hline & $\begin{array}{l}\text { Respondents with } \\
\text { axSpA }\end{array}$ & $\begin{array}{l}\text { Respondents } \\
\text { with PsA }\end{array}$ & $\begin{array}{l}\text { Respondents } \\
\text { with RA }\end{array}$ & $\begin{array}{l}\text { All respon- } \\
\text { dents }\end{array}$ \\
\hline \multicolumn{5}{|l|}{ Highest level of education, $n$ (\%) } \\
\hline $\mathrm{n}$ & 112 & 103 & 108 & 323 \\
\hline No formal education & 0 & I ( $(1.0)$ & 0 & $\mathrm{I}(0.3)$ \\
\hline Primary school education or less & I (0.9) & $3(2.9)$ & $2(1.9)$ & $6(1.9)$ \\
\hline $\begin{array}{l}\text { Secondary school education up to general, ordinary, or } \\
\text { standard level }\end{array}$ & $25(22.3)$ & $27(26.2)$ & $20(18.5)$ & $72(22.3)$ \\
\hline $\begin{array}{l}\text { Secondary school education up to intermediate, advanced, } \\
\text { or higher level }\end{array}$ & $14(12.5)$ & $18(17.5)$ & $14(13.0)$ & $46(14.2)$ \\
\hline $\begin{array}{l}\text { Professional or work-related qualifications obtained from a } \\
\text { college or university }\end{array}$ & $26(23.2)$ & $23(22.3)$ & $30(27.8)$ & $79(24.5)$ \\
\hline Undergraduate university degree & $22(19.6)$ & $12(11.7)$ & $27(25.0)$ & $61(18.9)$ \\
\hline Postgraduate university degree or equivalent qualification & $20(17.9)$ & II (I0.7) & $14(13.0)$ & $45(13.9)$ \\
\hline Prefer not to answer & $4(3.6)$ & $8(7.8)$ & $I(0.9)$ & $13(4.0)$ \\
\hline \multicolumn{5}{|l|}{ Employment status, n (\%) } \\
\hline $\mathrm{n}$ & 112 & 103 & 108 & 323 \\
\hline Employed full-time & $44(39.3)$ & $33(32.0)$ & $35(32.4)$ & $112(34.7)$ \\
\hline Employed part-time & $15(13.4)$ & $23(22.3)$ & $16(14.8)$ & $54(16.7)$ \\
\hline Self-employed & $7(6.3)$ & $7(6.8)$ & $7(6.5)$ & $21(6.5)$ \\
\hline Homemaker & $3(2.7)$ & $5(4.9)$ & $3(2.8)$ & II (3.4) \\
\hline Student & $5(4.5)$ & $2(1.9)$ & $4(3.7)$ & II (3.4) \\
\hline Retired & $21(18.8)$ & $15(14.6)$ & $20(18.5)$ & $56(17.3)$ \\
\hline Disabled/unable to work & $9(8.0)$ & $10(9.7)$ & $14(13.0)$ & $33(10.2)$ \\
\hline Unemployed but looking for work & $5(4.5)$ & $5(4.9)$ & $4(3.7)$ & $14(4.3)$ \\
\hline Unemployed and not looking for work & 0 & 0 & $2(1.9)$ & $2(0.6)$ \\
\hline Prefer not to answer & $3(2.7)$ & $3(2.9)$ & $3(2.8)$ & $9(2.8)$ \\
\hline \multicolumn{5}{|l|}{$\begin{array}{l}\text { Household income before tax and other deductions in } \\
2016, \mathrm{n}(\%)\end{array}$} \\
\hline $\mathrm{n}$ & 112 & 103 & 108 & 323 \\
\hline Less than $£ 25,000$ & $30(26.8)$ & $26(25.2)$ & $22(20.4)$ & $78(24.1)$ \\
\hline$£ 25,000$ to $£ 49,999$ & $29(25.9)$ & $30(29.1)$ & 31 (28.7) & $90(27.9)$ \\
\hline$£ 50,000$ to $£ 74,999$ & $17(15.2)$ & $14(13.6)$ & $22(20.4)$ & $53(16.4)$ \\
\hline$£ 75,000$ to $£ 99,999$ & $7(6.3)$ & $10(9.7)$ & $7(6.5)$ & $24(7.4)$ \\
\hline$f 100,000$ to $f 149,999$ & $3(2.7)$ & I (I.0) & $\mathrm{I}(0.9)$ & $5(1.5)$ \\
\hline$f 150,000$ to $f 199,999$ & I (0.9) & 0 & 0 & $\mathrm{I}(0.3)$ \\
\hline$£ 200,000$ or more & 0 & 0 & 0 & 0 \\
\hline Don't know/not sure & $6(5.4)$ & $4(3.9)$ & $6(5.6)$ & $16(5.0)$ \\
\hline Prefer not to answer & $19(17.0)$ & $18(17.5)$ & $19(17.6)$ & $56(17.3)$ \\
\hline
\end{tabular}

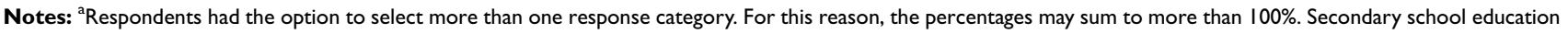
up to general, ordinary, or standard level: CSEs, GCSEs, O-levels, Scottish Standard Grades, or equivalent. Secondary school education up to intermediate, advanced, or higher level: A/S levels, A-levels, Scottish Intermediates, Scottish Highers, International/Scottish Baccalaureate or equivalent; Professional or work-related qualifications obtained from a college or university: Certificate of Higher Education, Diploma of Higher Education, foundation degree or equivalent; Undergraduate university degree: BSc, BA or equivalent; Postgraduate university degree or equivalent qualification: PGCE, MSc, MA, MPhil, PhD, or equivalent.

Abbreviations: axSpA, axial spondyloarthritis; COPD, chronic obstructive pulmonary disorder; EU, European Union; PsA, psoriatic arthritis; RA, rheumatoid arthritis; SD, standard deviation.

low, medium, and high. Therefore, the data from both cost ranges were pooled for the remaining analyses.

Table 4 presents results from the RPL model estimation. On average, respondents preferred self-injection devices with enhanced features over a standard self-injection. However, respondents did not show a statistically significant preference for a large or small grip size. The order of importance for each attribute can be extrapolated from the preference estimated parameters as all attributes could assume only two values (yes/no or large/small). The skin sensor was the most 
Table 3 Patients stratified by disease indication

\begin{tabular}{|c|c|c|c|}
\hline & $\begin{array}{l}\text { axSpA } \\
n=I 12\end{array}$ & $\begin{array}{l}\text { PsA } \\
n=103\end{array}$ & $\begin{array}{l}\text { RA } \\
n=108\end{array}$ \\
\hline \multicolumn{4}{|l|}{$\begin{array}{l}\text { Physician-assessed disease } \\
\text { severity, n (\%) }\end{array}$} \\
\hline Moderate & $51(45.5)$ & $53(51.5)$ & $57(52.8)$ \\
\hline Severe & $6 I(54.5)$ & $50(48.5)$ & $51(47.2)$ \\
\hline \multicolumn{4}{|l|}{$\begin{array}{l}\text { Self-injection experience, } \\
\text { n (\%) }\end{array}$} \\
\hline Self-injection experienced & $6 I(54.5)$ & $53(5 \mid .5)$ & $58(53.7)$ \\
\hline Self-injection naïve & $51(45.5)$ & $50(48.5)$ & $50(46.3)$ \\
\hline
\end{tabular}

Abbreviations: axSpA, axial spondyloarthritis; PsA, psoriatic arthritis; RA, rheumatoid arthritis.

important, with the highest preference weight for inclusion in the device, followed by electronic log, injection speed control and injection date reminder, step-by-step on-screen instructions, and grip size.

\section{Subgroup analysis}

When comparing a reusable self-injection device without any enhanced features and the standard disposable self- injection device, axSpA respondents had a statistically significant preference for a disposable standard self-injection device, while RA and PsA did not have a strong preference between the standard disposable device and a reusable device (Table 5). Patients with physician-assessed severe condition (across all indications) significantly preferred a disposable standard self-injection device, while respondents with physician-assessed moderate condition significantly preferred a reusable device (Table 6). Patients with self-injection experience were significantly more likely to select the disposable standard self-injection device, while self-injection naïve respondents had no preference (Table 7).

On average, respondents in each subgroup significantly preferred self-injection devices enhanced with any of the features included in the DCE, excluding grip size, which varied across subgroups. Specifically, respondents with axSpA significantly preferred a small grip, while PsA significantly preferred a large grip (Table 5). Respondents with moderate condition (across all conditions) significantly preferred a small grip, while respondents with severe condition

Table 4 Random parameters logit estimates $(\mathrm{N}=323)$

\begin{tabular}{|c|c|c|c|c|}
\hline \multirow{2}{*}{\multicolumn{2}{|c|}{$\begin{array}{l}\text { Attribute } \\
\text { Skin sensor }\end{array}$}} & \multirow{2}{*}{$\begin{array}{l}\text { Preference weight } \\
0.725^{* *}\end{array}$} & \multicolumn{2}{|c|}{$95 \%$ confidence interval } \\
\hline & & & 0.552 & 0.897 \\
\hline \multicolumn{2}{|c|}{ Control of the speed of injection } & $0.455^{* *}$ & 0.320 & 0.591 \\
\hline \multicolumn{2}{|c|}{ Step-by-step, on-screen instructions in real time } & $0.304 * *$ & 0.182 & 0.427 \\
\hline \multicolumn{2}{|c|}{ Injection date reminder } & $0.438 * *$ & 0.292 & 0.584 \\
\hline \multicolumn{2}{|l|}{ Electronic log } & $0.497 * *$ & 0.369 & 0.626 \\
\hline \multicolumn{2}{|l|}{ Large grip size } & -0.181 & -0.424 & 0.062 \\
\hline \multirow[t]{8}{*}{ Out-of-pocket cost } & $£ 0$ & $4.297 * *$ & 3.472 & 5.122 \\
\hline & $€ 15$ & $3.57 I^{* *}$ & 2.885 & 4.256 \\
\hline & $£ 30$ & $2.844 * *$ & 2.298 & 3.391 \\
\hline & $£ 50$ & $1.876 * *$ & 1.516 & 2.236 \\
\hline & $£ 90$ & $-0.06 I^{* *}$ & -0.072 & -0.049 \\
\hline & $€ 125$ & $-1.755 * *$ & -2.092 & -1.418 \\
\hline & $£ 150$ & $-2.965^{* *}$ & -3.535 & -2.396 \\
\hline & $£ 250$ & $-7.807^{* *}$ & -9.306 & -6.308 \\
\hline \multicolumn{2}{|c|}{ Constant for standard disposable self-injection device } & 0.338 & -0.286 & 0.961 \\
\hline
\end{tabular}

Notes: All variables, with the exception of out-of-pocket cost, were dichotomous and effects-coded. We present coefficients estimates for the included features, the coefficient for excluded features is computed as the negative of it. Cost was included in the model as the interaction of continuous cost and the natural log of income. The preference weights were created using the estimated coefficient for cost. The parameters were modeled with normally distributed random parameters. An error component was included to better accommodate the opt-out bias that could be caused by the standard device alternative. $* * P \leq 0.01$. 
Table 5 Random parameters logit regression results: condition subgroups ( $N=323)$

\begin{tabular}{|c|c|c|c|c|}
\hline \multicolumn{2}{|l|}{ Attribute } & Preference weight & \multicolumn{2}{|c|}{$95 \%$ confidence interval } \\
\hline \multicolumn{5}{|l|}{ axSpA Subgroup } \\
\hline \multicolumn{2}{|l|}{ Skin sensor } & $0.988 * *$ & 0.678 & 1.297 \\
\hline \multicolumn{2}{|c|}{ Control of the speed of injection } & $0.659 * *$ & 0.428 & 0.891 \\
\hline \multicolumn{2}{|c|}{ Step-by-step, on-screen instructions in real time } & $0.235^{*}$ & 0.011 & 0.459 \\
\hline \multicolumn{2}{|c|}{ Injection date reminder } & $0.733 * *$ & 0.465 & 1.000 \\
\hline \multicolumn{2}{|l|}{ Electronic log } & $0.655^{* *}$ & 0.417 & 0.892 \\
\hline \multicolumn{2}{|l|}{ Large grip size } & $-0.73 I^{* *}$ & -1.136 & -0.325 \\
\hline \multirow[t]{8}{*}{ Out-of-pocket cost } & $€ 0$ & $4.855^{* *}$ & 3.849 & 5.862 \\
\hline & $£ 15$ & $4.035 * *$ & 3.198 & 4.871 \\
\hline & $£ 30$ & $3.214 * *$ & 2.548 & 3.881 \\
\hline & $£ 50$ & $2.120 * *$ & 1.680 & 2.560 \\
\hline & $£ 90$ & $-0.068 * *$ & -0.083 & -0.054 \\
\hline & $£ \mid 25$ & $-1.983 * *$ & -2.394 & -1.572 \\
\hline & $£ 150$ & $-3.35 I^{* *}$ & -4.046 & -2.656 \\
\hline & $£ 250$ & $-8.822 * *$ & $-|0.65|$ & -6.993 \\
\hline \multicolumn{2}{|c|}{ Constant for standard disposable self-injection device } & $1.681 * *$ & 0.624 & 2.739 \\
\hline \multicolumn{5}{|l|}{ PsA Subgroup } \\
\hline \multicolumn{2}{|l|}{ Skin sensor } & $0.773 * *$ & 0.451 & 1.096 \\
\hline \multicolumn{2}{|c|}{ Control of the speed of injection } & $0.442 * *$ & 0.216 & 0.668 \\
\hline \multicolumn{2}{|c|}{ Step-by-step, on-screen instructions in real time } & $0.402 * *$ & 0.169 & 0.635 \\
\hline \multicolumn{2}{|c|}{ Injection date reminder } & $0.444 * *$ & 0.206 & 0.681 \\
\hline \multicolumn{2}{|l|}{ Electronic log } & $0.476 * *$ & 0.257 & 0.694 \\
\hline \multicolumn{2}{|l|}{ Large grip size } & $0.516 * *$ & 0.128 & 0.904 \\
\hline \multirow[t]{8}{*}{ Out-of-pocket cost } & $£ 0$ & $4.892 * *$ & 3.617 & 6.166 \\
\hline & 615 & $4.065 * *$ & 3.006 & 5.124 \\
\hline & $£ 30$ & $3.238 * *$ & 2.395 & 4.082 \\
\hline & $£ 50$ & $2.136 * *$ & 1.579 & 2.692 \\
\hline & $£ 90$ & $-0.069 * *$ & -0.087 & -0.051 \\
\hline & $£ \mid 25$ & $-1.998 * *$ & -2.518 & -1.477 \\
\hline & $£ 150$ & $-3.376 * *$ & -4.255 & -2.496 \\
\hline & $£ 250$ & $-8.888 * *$ & -11.203 & -6.572 \\
\hline \multicolumn{2}{|c|}{ Constant for standard disposable self-injection device } & 0.457 & -0.564 & 1.478 \\
\hline
\end{tabular}


Table 5 (Continued).

\begin{tabular}{|c|c|c|c|c|}
\hline \multicolumn{2}{|l|}{ Attribute } & Preference weight & \multicolumn{2}{|c|}{$95 \%$ confidence interval } \\
\hline \multicolumn{5}{|l|}{ RA Subgroup } \\
\hline \multicolumn{2}{|l|}{ Skin sensor } & $0.589 * *$ & 0.303 & 0.875 \\
\hline \multicolumn{2}{|c|}{ Control of the speed of injection } & $0.577^{* *}$ & 0.342 & 0.812 \\
\hline \multicolumn{2}{|c|}{ Step-by-step, on-screen instructions in real time } & $0.280 * *$ & 0.069 & 0.491 \\
\hline \multicolumn{2}{|c|}{ Injection date reminder } & $0.480 * *$ & 0.237 & 0.723 \\
\hline \multicolumn{2}{|l|}{ Electronic log } & $0.433^{* *}$ & 0.204 & 0.662 \\
\hline \multicolumn{2}{|l|}{ Large grip size } & 0.193 & -0.235 & 0.622 \\
\hline \multirow[t]{8}{*}{ Out-of-pocket cost } & E0 & $3.708^{* *}$ & 2.905 & 4.512 \\
\hline & $€ 15$ & $3.082 * *$ & 2.414 & 3.750 \\
\hline & $£ 30$ & $2.455^{* *}$ & 1.923 & 2.987 \\
\hline & $£ 50$ & $1.619 * *$ & 1.268 & 1.970 \\
\hline & $£ 90$ & $-0.052 * *$ & -0.064 & -0.041 \\
\hline & E125 & $-1.515^{* *}$ & -1.843 & -1.186 \\
\hline & 6150 & $-2.559 * *$ & -3.114 & -2.005 \\
\hline & $£ 250$ & $-6.738 * *$ & -8.198 & -5.277 \\
\hline \multicolumn{2}{|c|}{ Constant for standard disposable self-injection device } & -0.933 & -2.757 & 0.891 \\
\hline
\end{tabular}

Notes: All variables, with the exception of out-of-pocket cost, were dichotomous and effects-coded. Cost was included in the model as the interaction of continuous cost and the natural log of income. The preference weights in the table above have been created using the estimated coefficient for cost. Dummy-coded variables for PsA and RA were interacted with the main variables to model the subgroups. The parameters were modeled with normally distributed random parameters, assumed to be the same across the axSpA, PsA, and RA subgroups. Additionally, we included an error component to better accommodate the opt-out bias that could be caused by the standard device alternative. $* * P \leq 0.01 ; * P \leq 0.05$.

Abbreviations: axSpA, axial spondyloarthritis; PsA, psoriatic arthritis; RA, rheumatoid arthritis.

qualitatively preferred a large grip; however, preference weights were not statistically significant (Table 6).

Further post-hoc subgroup analyses were carried out using a Wald test for systematic differences in preferences to investigate if age or gender influenced patient preference and if there were any significant differences compared to the overall population; however, no differences were found.

\section{Welfare analysis}

Despite residing in a health care system where the majority of treatment is free at the point of use, patients were willing to pay for an enhanced device. Patients were willing to pay for each different additional feature, with most to least valued being skin sensor (£30), injection speed control, injection reminders, electronic $\log (\sim £ 20$ each), on-screen instructions $(\sim £ 12)$, and grip size ( $£ 7)$ (Figure 2). Similar results were observed when patients were analyzed by subgroup with patients willing to pay for any of the enhanced features, except for grip size (Figure 3A-C).

When all enhanced features were considered together in a single hypothetical device (ie, calculating $\mathrm{CV}$ [Supplementary Materials]), all patients and all subgroups of patients valued the device over a standard disposable device (Figure 4). On average, respondents were willing to pay $£ 85$ for a reusable device with all enhanced features. However, there were variations in the amount patients were willing to pay for an enhanced device. Self-injection naïve and RA respondents were willing to pay the most ( $£ 130$ and $\sim £ 145$, respectively), whereas patients with self-injection experience were only willing to pay $£ 35$ for the same hypothetical device.

\section{Discussion}

Self-injection can offer convenience and independence for patients; however, factors associated with self-injection and 
Table 6 Random parameters logit regression results: disease severity subgroups $(\mathrm{N}=323)$

\begin{tabular}{|c|c|c|c|c|}
\hline \multicolumn{2}{|l|}{ Attribute } & Preference weight & \multicolumn{2}{|c|}{$95 \%$ confidence interval } \\
\hline \multicolumn{5}{|c|}{ Severe disease subgroup } \\
\hline \multicolumn{2}{|l|}{ Skin sensor } & $0.953^{* *}$ & 0.697 & 1.210 \\
\hline \multicolumn{2}{|c|}{ Control of the speed of injection } & $0.529 * *$ & 0.324 & 0.734 \\
\hline \multicolumn{2}{|c|}{ Step-by-step, on-screen instructions in real time } & $0.331 * *$ & 0.122 & 0.540 \\
\hline \multicolumn{2}{|c|}{ Injection date reminder } & $0.474 * *$ & 0.265 & 0.684 \\
\hline \multicolumn{2}{|l|}{ Electronic log } & $0.512 * *$ & 0.311 & 0.714 \\
\hline \multicolumn{2}{|l|}{ Large grip size } & 0.145 & -0.220 & 0.510 \\
\hline \multirow[t]{8}{*}{ Out-of-pocket cost } & ŁO & $5.098 * *$ & 4.188 & 6.008 \\
\hline & $£ 15$ & $4.236 * *$ & 3.480 & 4.992 \\
\hline & $£ 30$ & $3.375^{* *}$ & 2.773 & 3.977 \\
\hline & $£ 50$ & $2.226 * *$ & 1.829 & 2.623 \\
\hline & $£ 90$ & $-0.072^{* *}$ & -0.085 & -0.059 \\
\hline & $€ \mid 25$ & $-2.082 * *$ & -2.454 & -1.711 \\
\hline & $£ \mid 50$ & $-3.518^{* *}$ & -4.146 & -2.891 \\
\hline & $£ 250$ & $-9.263 * *$ & -10.916 & -7.610 \\
\hline \multicolumn{2}{|c|}{ Constant for standard disposable self-injection device } & $1.474 * *$ & 0.466 & 2.481 \\
\hline \multicolumn{5}{|c|}{ Moderate disease subgroup } \\
\hline \multicolumn{2}{|l|}{ Skin sensor } & $0.566 * *$ & 0.341 & 0.790 \\
\hline \multicolumn{2}{|c|}{ Control of the speed of injection } & $0.422^{* *}$ & 0.249 & 0.594 \\
\hline \multicolumn{2}{|c|}{ Step-by-step, on-screen instructions in real time } & $0.299 * *$ & 0.126 & 0.472 \\
\hline \multicolumn{2}{|c|}{ Injection date reminder } & $0.610^{* *}$ & $0.41 \mathrm{I}$ & 0.810 \\
\hline \multicolumn{2}{|l|}{ Electronic log } & $0.491 * *$ & 0.313 & 0.669 \\
\hline \multicolumn{2}{|l|}{ Large grip size } & $-0.458 * *$ & -0.787 & -0.128 \\
\hline \multirow[t]{8}{*}{ Out-of-pocket cost } & $£ 0$ & $4.286 * *$ & 3.488 & 5.084 \\
\hline & 615 & $3.562^{* *}$ & 2.899 & 4.225 \\
\hline & $£ 30$ & $2.837^{* *}$ & 2.309 & 3.366 \\
\hline & $£ 50$ & $\left.1.87\right|^{* *}$ & 1.523 & 2.220 \\
\hline & $£ 90$ & $-0.060 * *$ & -0.072 & -0.049 \\
\hline & $£ 125$ & $-1.75 I^{* *}$ & -2.077 & -1.425 \\
\hline & $£ \mid 50$ & $-2.958^{* *}$ & -3.509 & -2.407 \\
\hline & $£ 250$ & $-7.788 * *$ & -9.238 & -6.338 \\
\hline \multicolumn{2}{|c|}{ Constant for standard disposable self-injection device } & $-0.82 I^{*}$ & -1.574 & -0.067 \\
\hline
\end{tabular}

Notes: All variables, with the exception of out-of-pocket cost, were dichotomous and effects-coded. Cost was included in the model as the interaction of continuous cost and the natural log of income. The preference weights in the table above have been created using the estimated coefficient for cost. A dummy-coded variable for moderate disease was interacted with the main variables to model the subgroups. The parameters were modeled with normally distributed random parameters, assumed to be the same across the disease severity subgroups. Additionally, we included an error component to better accommodate the opt-out bias that could be caused by the standard device alternative. ${ }^{* * P} \leq 0.01 ; * P \leq 0.05$. 
Table 7 Random parameters logit regression results: self-injection experience subgroups $(\mathrm{N}=323)$

\begin{tabular}{|c|c|c|c|c|}
\hline \multicolumn{2}{|l|}{ Attribute } & Preference weight & \multicolumn{2}{|c|}{$95 \%$ confidence interval } \\
\hline \multicolumn{5}{|c|}{ No self-injection experience subgroup } \\
\hline \multicolumn{2}{|l|}{ Skin sensor } & $0.755^{* *}$ & 0.535 & 0.975 \\
\hline \multicolumn{2}{|c|}{ Control of the speed of injection } & $0.555^{* *}$ & 0.361 & 0.749 \\
\hline \multicolumn{2}{|c|}{ Step-by-step, on-screen instructions in real time } & $0.372^{* *}$ & 0.204 & 0.540 \\
\hline \multicolumn{2}{|c|}{ Injection date reminder } & $0.446 * *$ & 0.254 & 0.638 \\
\hline \multicolumn{2}{|l|}{ Electronic log } & $0.529 * *$ & 0.346 & 0.712 \\
\hline \multicolumn{2}{|l|}{ Large grip size } & -0.153 & -0.474 & 0.169 \\
\hline \multirow[t]{8}{*}{ Out-of-pocket cost } & $£ 0$ & $3.758 * *$ & 3.024 & 4.492 \\
\hline & $£ \mid 5$ & $3.123 * *$ & 2.513 & 3.733 \\
\hline & $£ 30$ & $2.488 * *$ & 2.002 & 2.973 \\
\hline & $£ 50$ & $1.64 I^{* *}$ & 1.320 & 1.961 \\
\hline & $£ 90$ & $-0.053 * *$ & -0.063 & -0.043 \\
\hline & $£ 125$ & $-1.535 * *$ & -1.835 & -1.235 \\
\hline & 6150 & $-2.594 * *$ & -3.100 & -2.087 \\
\hline & $£ 250$ & $-6.828 * *$ & -8.161 & -5.494 \\
\hline \multicolumn{2}{|c|}{ Constant for standard disposable self-injection device } & -0.486 & -1.360 & 0.389 \\
\hline \multicolumn{5}{|c|}{ Self-injection experience subgroup } \\
\hline \multicolumn{2}{|l|}{ Skin sensor } & $0.679 * *$ & 0.382 & 0.976 \\
\hline \multicolumn{2}{|c|}{ Control of the speed of injection } & $0.470 * *$ & 0.222 & 0.717 \\
\hline \multicolumn{2}{|c|}{ Step-by-step, on-screen instructions in real time } & $0.278 * *$ & 0.072 & 0.484 \\
\hline \multicolumn{2}{|c|}{ Injection date reminder } & $0.778 * *$ & 0.526 & 1.029 \\
\hline \multicolumn{2}{|l|}{ Electronic log } & $0.46 I^{* *}$ & 0.237 & 0.685 \\
\hline \multicolumn{2}{|l|}{ Large grip size } & $-0.536 * *$ & $-0.87 \mid$ & -0.201 \\
\hline \multirow[t]{8}{*}{ Out-of-pocket cost } & $£ 0$ & $6.007^{* *}$ & 4.757 & 7.257 \\
\hline & 615 & $4.992 * *$ & 3.953 & 6.031 \\
\hline & $£ 30$ & $3.977 * *$ & 3.149 & 4.804 \\
\hline & $£ 50$ & $2.623 * *$ & 2.077 & 3.169 \\
\hline & $£ 90$ & $-0.085 * *$ & -0.102 & -0.067 \\
\hline & $€ 125$ & $-2.454 * *$ & -2.964 & -1.943 \\
\hline & $€ 150$ & $-4.146 * *$ & -5.009 & -3.283 \\
\hline & $£ 250$ & $-10.914 * *$ & -13.186 & -8.643 \\
\hline \multicolumn{2}{|c|}{ Constant for standard disposable self-injection device } & $1.882 * *$ & 0.615 & 3.148 \\
\hline
\end{tabular}

Notes: All variables, with the exception of out-of-pocket cost, were dichotomous and effects-coded. Cost was included in the model as the interaction of continuous cost and the natural log of income. The preference weights in the table above have been created using the estimated coefficient for cost. A dummy-coded variable for selfinjection experience was interacted with the main variables to model the subgroups. The parameters were modeled with normally distributed random parameters, assumed to be the same across the self-injection experience subgroups. Additionally, we included an error component to better accommodate the opt-out bias that could be caused by the standard device alternative. $* * P \leq 0.01$. 


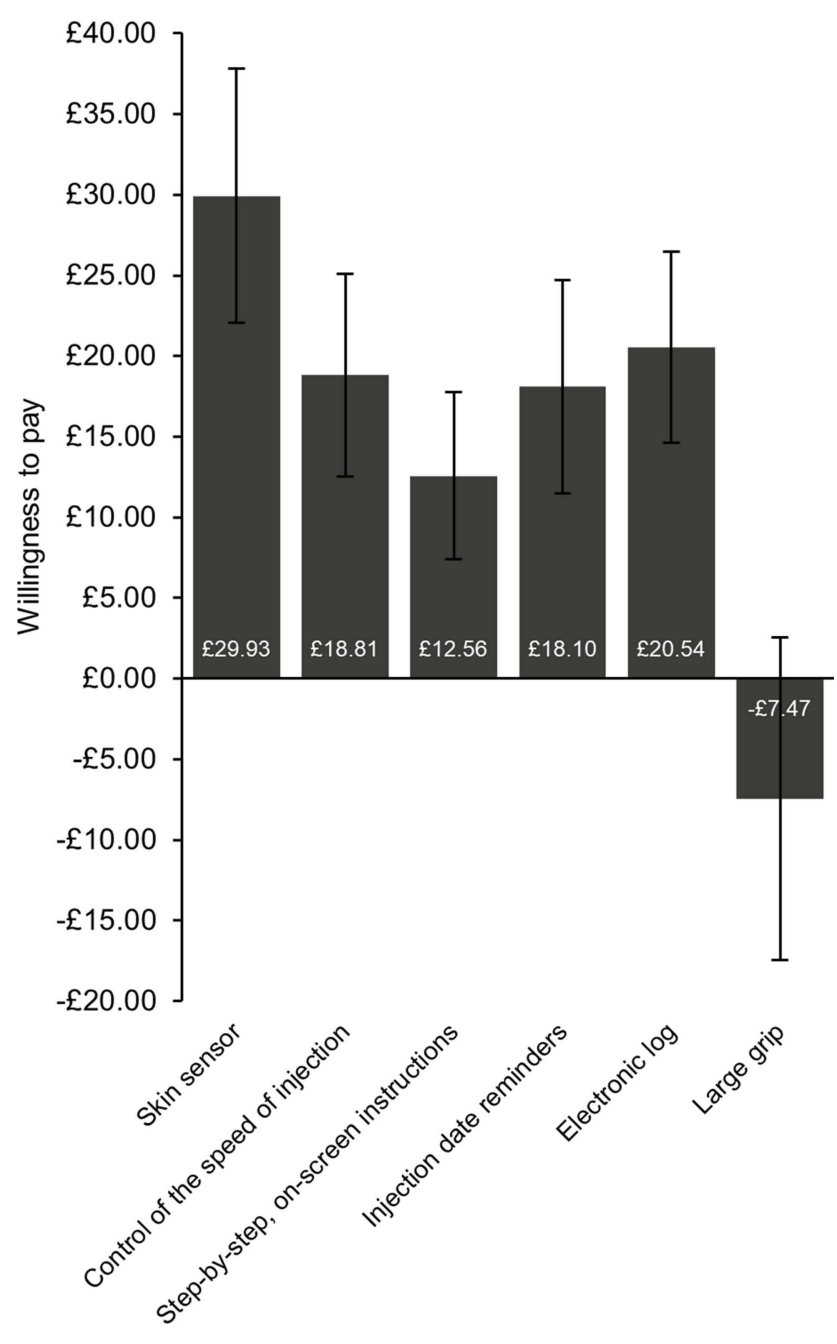

Figure 2 Total population willingness to pay for each enhanced feature $(\mathrm{N}=323)$.

treatment delivery can affect patient adherence to medication. ${ }^{3,4}$ Differences in patient subgroup preferences for certain device features have previously been reported. ${ }^{15,25}$ These results suggest that optimizing device design to meet patient needs, expectations and preferences may help to improve patient experience. ${ }^{1}$ The results of this study demonstrate that despite certain subgroups preferring a standard disposable device over a reusable device without enhanced features, all patients preferred a self-injection device with enhanced features over a device with no enhanced features. It is important to note that respondents were willing to pay for an enhanced device despite living in the UK, which has a health care system where patients typically do not pay or pay very little at the point of delivery for prescribed medication or medical devices. Carrying out the same DCE in a country in which residents pay more for their health care at the point of delivery may find respondents are willing to pay more for the same enhanced device.
Generally, all disease subgroups valued the skin sensor more than other enhanced features, while the on-screen instructions were not as highly valued by all disease subgroups. Differences in patient preference for grip size were observed; axSpA patients preferred a small grip size, PsA patients preferred a large grip size, and RA patients did not express a significant preference. The different preferences for grip size among patients may be caused by the impact the disease has on hand dexterity. axSpA is a condition that primarily affects the axial skeleton, whereas PsA and RA generally affect smaller joints, such as the finger, thumb, and wrist joints in the hands. ${ }^{26-28}$ The large grip was designed for patients who struggle with self-injection as they have trouble holding objects, so patients whose condition affects hand dexterity may express a preference for the large grip. RA patients did not show a statistically significant preference for the large grip despite often having hand dexterity problems. However, this may be due to RA patients with different levels of disease severity preferring different grip sizes. It may be that severe RA patients show a preference for a large grip, but RA patients with moderate disease do not.

In addition to differences in patient subgroup preferences for individual features, these results revealed differences in the willingness of patients to pay for a new device. Specifically, self-injection naïve patients were willing to pay more than self-injection experienced patients for a device with the same enhanced features. Previous studies have shown that self-injection naïve patients have more self-injection anxieties and need more support. ${ }^{18,29}$ Therefore, the higher WTP among naïve patients may be driven by the extra support (eg, step-by-step on-screen instructions) and certainty (eg, as given by the skin sensor) the enhanced device provides. In contrast, experienced patients are more familiar with self-injection and may have a routine, needing less assistance from a device to feel comfortable with the self-injection process. ${ }^{18}$ This is supported by the results showing that self-injection experienced patients and patients with moderate disease control are willing to pay less for every enhanced feature, compared to self-injection naïve patients.

The differences in patient preferences observed in our study support a targeted approach to device design and demonstrate that a choice of injection devices is important for patient satisfaction. A previous DCE study found that, while there is heterogeneity in RA patient treatment preference, patients generally fall into one of the five "preference" groups. Each preference group had a different top 


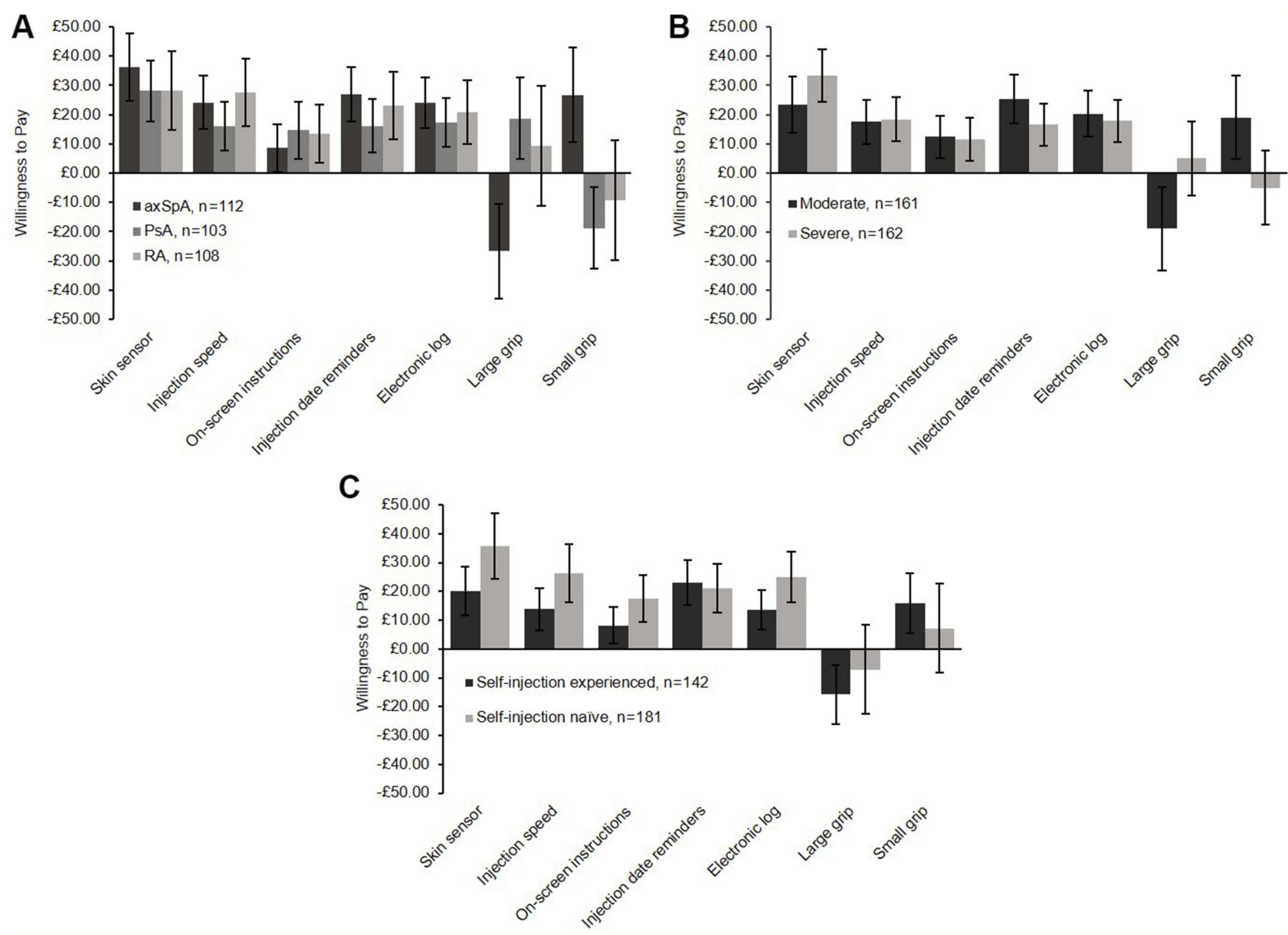

Figure 3 Population subgroup willingness to pay for each enhanced feature. (A) Willingness to pay for each enhanced feature by condition; (B) Willingness to pay for each enhanced feature by disease severity; (C) Willingness to pay to each enhanced feature by self-injection experience.

priority when deciding which treatment to use including avoiding side effects that impact quality of life, receiving fast-acting medicine, avoiding needles, avoiding life-threatening adverse events and treatment affordability. Determining which group a patient falls into could aid decisions about which self-injection device may best suit their priorities and help remove barriers to self-injection. ${ }^{30}$ Providing a portfolio of different self-injection devices could help meet the needs of multiple different "preference" groups and allow patients to choose a device that best fits their individual needs. For example, a patient with hand dexterity problems may prefer to use a device with a larger grip, while a patient without disease affecting their hands may prefer a device with a smaller grip.

Several previous studies have validated the use of DCE and WTP methodology in a rheumatology population. ${ }^{12,31,32}$ Both methods have been found to be reliable over time, and WTP has been shown to consistently assess symptom severity over time, suggesting the method has good construct validity. ${ }^{12,31}$ Overall, these studies support the use of the DCE and WTP methods to investigate patient preference.

\section{Limitations}

This study was associated with several limitations. There was the potential for self-selection bias as patients could decide whether to participate in the survey. Additionally, patients were recruited via a small number of physicians possibly introducing further selection bias as physicians could also choose to participate in the study. The attributes of the treatment alternatives presented in the survey mimicked those of real-world devices as closely as possible; however, they were hypothetical devices and so the results were subject to potential hypothetical bias. Patients were not able to handle the devices, so it may be more difficult for respondents to evaluate features such as grip size. Not all attributes of a device were included in the survey, and the results from this study related only to attributes and attribute levels defined in the survey. Attributes were chosen based on 


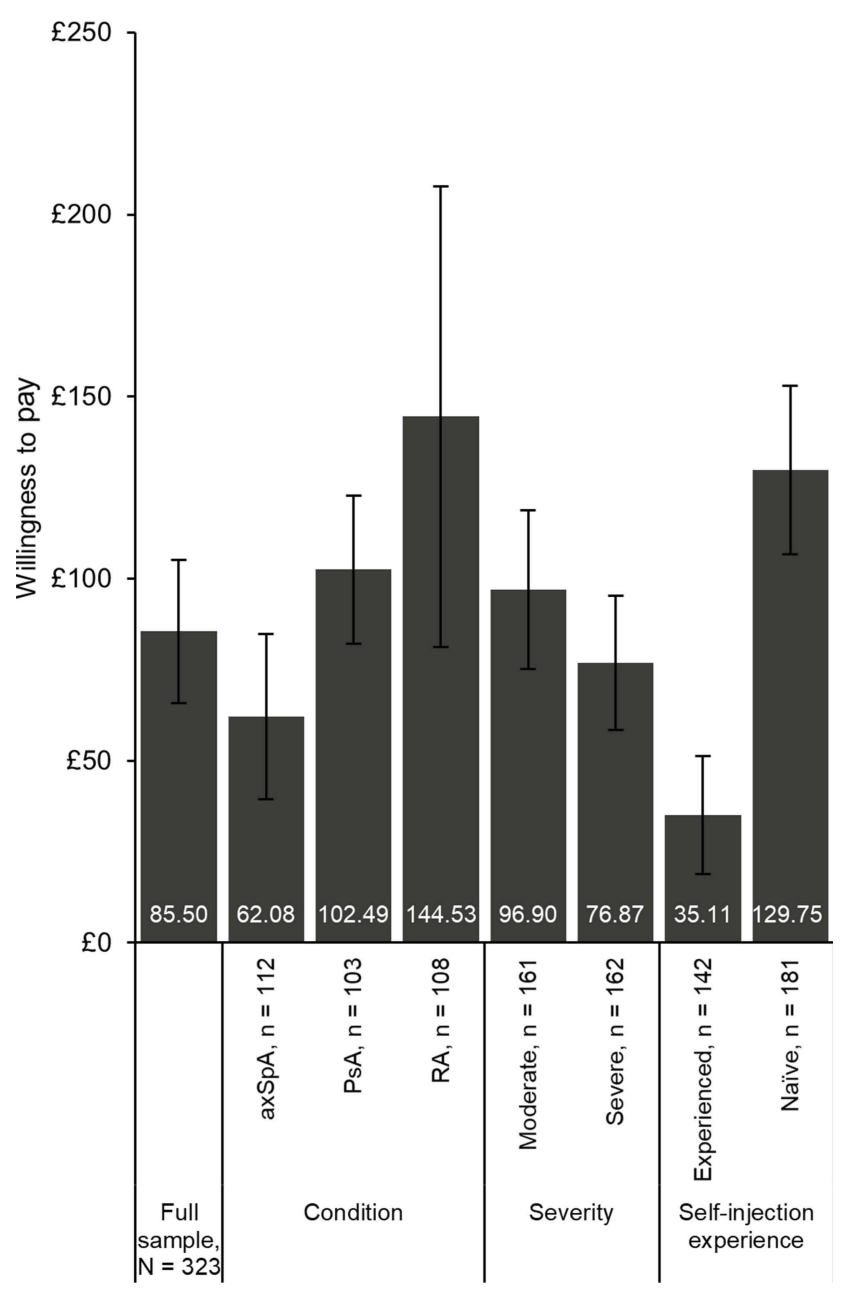

Figure 4 Sample and subgroup valuation of an enhanced, reusable self-injection device with all enhanced features and large grip compared to a standard disposable device $(\mathrm{N}=323)$. A standard disposable self-injection device has no features, small grip, and no out-of-pocket cost associated with it.

the opinions of health care professionals (HCPs) at an advisory board and on patient preferences in previous studies. ${ }^{17,18}$ It is possible that this could have introduced bias to the attributes included, as choice was partly patientdriven and partly driven by the opinions of HCPs. In the UK, patients do not usually pay for medical devices which limits the generalizability of the results. For example, if the study was conducted in the US, where patients pay for their health care, patients might be willing to pay more for additional features. Despite this limitation, a positive WTP for different enhanced features, above what respondents stated they have paid for medical devices in the past, was found. Finally, it should be noted that treatment non-adherence has a variety of causes (not just treatment delivery factors) ${ }^{1}$ and that interventions to improve adherence have previously been shown to have modest effectiveness. ${ }^{33}$ Additional evidence may therefore be required to confirm the real-world impact of a reusable self-injection device with enhanced features on patient treatment adherence.

\section{Conclusion}

Patients positively valued and were therefore willing to pay for reusable self-injection devices with enhanced features, compared to a disposable self-injection device with no enhanced features. This suggests that patient experience may be improved by a self-injection device with these enhanced features and that current self-injection devices may not meet all needs of patients with RA, PsA, and axSpA. A greater choice of injection devices, which could include enhanced features, may improve patient experience, satisfaction and treatment adherence and, therefore, treatment outcomes.

\section{Acknowledgments}

The authors thank the patients, the investigators, and their teams who took part in this study. The authors would also like to thank Kimberly Moon for her help in project management. The authors also acknowledge Susanne Wiegratz, UCB Pharma, Monheim am Rhein, Germany for publication coordination and Emma Phillips, $\mathrm{PhD}$, and Simon Foulcer, $\mathrm{PhD}$, from Costello Medical, UK, for medical writing and editorial assistance in preparing this manuscript for publication, based on the authors' input and direction. This study was funded by UCB Pharma. The abstract of this paper was presented at the International Society for Pharmacoeconomics and Outcomes Research (ISPOR) Europe 2018 Conference as a conference talk with interim findings. The talk's abstract was published in ISPOR Europe 2018 Research Abstracts in Value in Health 2018;21(S3): https://doi.org/10.1016/j.jval.2018.09.058.

\section{Disclosure}

MB: employee of RTI; BS: employee of UCB Pharma; BH: employee of RTI; BM: employee of RTI; IM: employee of UCB Pharma; MS: consultancy fees from UCB Pharma; NM: consultancy fees from UCB Pharma. The authors report no other conflicts of interest in this work.

\section{References}

1. Domanska B, Stumpp O, Poon S, Oray S, Mountian I, Pichon C. Using patient feedback to optimize the design of a certolizumab pegol electromechanical self-injection device: insights from human factors studies. Adv Ther. 2018;35(1):100-115. doi:10.1007/s12325017-0645-1 
2. Marengo MF, Suarez-Almazor ME. Improving treatment adherence in patients with rheumatoid arthritis: what are the options? Int J Clin Rheumtol. 2015;10(5):345-356. doi:10.2217/ijr.15.39

3. Anderson BJ, Redondo MJ. What can we learn from patient-reported outcomes of insulin pen devices? J Diabetes Sci Technol. 2011;5 (6):1563-1571. doi:10.1177/193229681100500633

4. Schwartzman S, Morgan GJ Jr. Does route of administration affect the outcome of TNF antagonist therapy? Arthritis Res Ther. 2004;6 (Suppl 2):S19-S23. doi:10.1186/ar996

5. Keininger D, Coteur G. Assessment of self-injection experience in patients with rheumatoid arthritis: psychometric validation of the Self-Injection Assessment Questionnaire (SIAQ). Health Qual Life Outcomes. 2011;9:2. doi:10.1186/1477-7525-9-40

6. Bailey T, Thurman J, Niemeyer M, Schmeisl G. Usability and preference evaluation of a prefilled insulin pen with a novel injection mechanism by people with diabetes and healthcare professionals. Curr Med Res Opin. 2011;27(10):2043-2052. doi:10.1185/03007995.2011.616190

7. Sheikhzadeh A, Yoon J, Formosa D, Domanska B, Morgan D, Schiff M. The effect of a new syringe design on the ability of rheumatoid arthritis patients to inject a biological medication. Appl Ergon. 2012;43(2):368-375. doi:10.1016/j.apergo.2011.05.014

8. Domanska B, VanLunen B, Peterson L, Mountian I, Schiff M. Comparative usability study for a certolizumab pegol autoinjection device in patients with rheumatoid arthritis. Expert Opin Drug Deliv. 2017;14(1):15-22. doi:10.1080/17425247.2016.1256283

9. Gossec L, Berenbaum F, Chauvin P, et al. Development and application of a questionnaire to assess patient beliefs in rheumatoid arthritis and axial spondyloarthritis. Clin Rheumatol. 2018;37(10):2649-2657. doi: 10.1007/s10067-018-4172-5

10. Gossec L, Chauvin P, Saraux A, et al. Development and psychometric validation of a patient-reported outcome measure to assess fears in rheumatoid arthritis and axial spondyloarthritis: the Fear Assessment in Inflammatory Rheumatic diseases (FAIR) questionnaire. Ann Rheum Dis. 2018;77(2):258-263. doi:10.1136/annrheumdis-2017212000

11. Lancsar E, Louviere J. Conducting discrete choice experiments to inform healthcare decision making: a user's guide. PharmacoEconomics. 2008;26(8):661-677. doi:10.2165/00019053-200826080-00004

12. Skjoldborg US, Lauridsen J, Junker P. Reliability of the discrete choice experiment at the input and output level in patients with rheumatoid arthritis. Value Health. 2009;12(1):153-158. doi:10.1111/j.15244733.2008.00402.x

13. Dilla T, Rentero ML, Comellas M, Lizan L, Sacristan JA. Patients' preferences for rheumatoid arthritis treatments and their participation in the treatment decision-making process. A systematic review of the literature. Value Health. 2015;18(7):A652. doi:10.1016/j. jval.2015.09.2348

14. UCB: UCB receives positive CHMP opinion for CIMZIA ${ }^{\circledR}$ (certolizumab pegol) dose dispenser cartridge for use with ava ${ }^{\circledR}$ electronic injection device. Available from: https:/www.ucb.com/stories-media/ Press-Releases/article/UCB-receives-positive-CHMP-opinion-forCIMZIA-certolizumab-pegol-dose-dispenser-cartridge-for-use-withava-electronic-injection-device. accessed April, 2018.

15. Collier DH, Bitman B, Coles A, Liu L, Kumar S, Judd C. A novel electromechanical autoinjector, AutoTouch, for self-injection of etanercept: real-world use and benefits. Postgrad Med. 2017;129 (1):118-125. doi:10.1080/00325481.2017.1251291
16. Bridges JFP, Hauber AB, Marshall D, et al. Conjoint analysis applications in health - a checklist: a report of the ISPOR good research practices for conjoint analysis task force. Value Health. 2011;14 (4):403-413. doi:10.1016/j.jval.2010.11.013

17. Domańska B, Mountian I, Vinconneau G. Patient-preferred design features of TNF inhibitor self-injection devices: insights from a rheumatoid arthritis auto-injector preference study. Value Health. 2017;20(9):A591-A592. doi:10.1016/j.jval.2017.08.1095

18. Schiff M, Saunderson S, Mountian I, Hartley P. Chronic disease and self-injection: ethnographic investigations into the patient experience during treatment. Rheumatol Ther. 2017;4(2):445-463. doi:10.1007/ s40744-017-0080-4

19. Ozdemir S, Johnson FR, Hauber AB. Hypothetical bias, cheap talk, and stated willingness to pay for health care. J Health Econ. 2009;28 (4):894-901. doi:10.1016/j.jhealeco.2009.04.004

20. Kuhfeld WF. Marketing Research Methods in SAS Experimental Design, Choice, Conjoint, and Graphical Techniques. Cary: SAS Institute Inc.; 2009.

21. Hauber AB, Gonzalez JM, Groothuis-Oudshoorn CG, et al. Statistical methods for the analysis of discrete choice experiments: a report of the ISPOR conjoint analysis good research practices task force. Value Health. 2016;19(4):300-315. doi:10.1016/j.jval.2016.04.004

22. Walker JL, Ben-Akiva M, Bolduc D. Identification of parameters in normal error component logit-mixture (NECLM) models. $J$ Appl Econom. 2007;22(6):1095-1125. doi:10.1002/(ISSN)1099-1255

23. Greene W. Econometric Analysis. Essex: Pearson Education Limited; 2012:155-161.

24. Hanemann WM. Welfare evaluations in contingent valuation experiments with discrete responses. Am J Agric Econ. 1984;66(3):332341. doi: $10.2307 / 1240800$

25. Kruger K, Alten R, Schiffner-Rohe J, et al. Patient preferences in the choice of disease modifying anti-rheumatic drugs. Ann Rheum Dis. 2015;74(suppl 2):322. doi:10.1136/annrheumdis-2015-eular.2349

26. UCB. Axial spondyloarthritis. 2017. Available from: https:/www. ucb.com/disease-areas/axial-spondyloarthritis. accessed June 2018]

27. UCB. Rheumatoid arthritis. 2017. Available from: https://www.ucb. com/disease-areas/rheumatoid-arthritis accessed June, 2018.

28. UCB. Psoriatic arthritis. 2017. Available from: https://www.ucb.com/ disease-areas/psoriatic-arthritis. accessed June, 2018.

29. Matfin G, Van Brunt K, Zimmermann AG, Threlkeld R, Ignaut DA. Safe and effective use of the once weekly dulaglutide single-dose pen in injection-naïve patients with type 2 diabetes. J Diabetes Sci Technol. 2015;9 (5):1071-1079. doi:10.1177/1932296815583059

30. Fraenkel L, Wiedmeyer C, Herath G, Michel G, Nowell B. Development of RA patient preference phenotypes [abstract]. Arthritis Rheumatol. 2016;68(suppl 10).

31. Tuominen R, Azbel M, Hemmila J, Mottonen T. Willingness to pay for improvement of physical function among rheumatoid arthritis patients as measured by health assessment questionnaire. Rheumatol Int. 2011;31(3):347-352. doi:10.1007/s00296-009-1280-1

32. Slothuus U, Brooks RG. Willingness to pay in arthritis: a Danish contribution. Rheumatology (Oxford). 2000;39(7):791-799. doi:10.1093/rheumatology/39.7.791

33. van den Bemt BJ, Zwikker HE, van den Ende CH. Medication adherence in patients with rheumatoid arthritis: a critical appraisal of the existing literature. Expert Rev Clin Immunol. 2012;8(4):337351. doi: $10.1586 /$ eci. 12.23 


\section{Supplementary materials \\ Discrete choice experiment}

Budget reminder, also referred to as "cheap talk" script

To ensure patients understood that the purpose of the survey was to elicit the value, and not the price of the device features presented, the following "cheap talk" script was included in the survey before the DCE questions.

Before you answer the following questions, we want to ask you to help us with a problem we have in studies like this one. Because people do not really have to pay the cost of the device they would choose, they often do not focus on the actual amounts shown, but only whether one cost is larger than another cost.

For example, if the costs of the devices in the questions are $£ 25, £ 50$, and $£ 100$, people often think of them as just "low," "medium," and "high." They do not really think about what they would have to give up — such as a restaurant meal or some new clothes - if they had to pay ( $£ 25$, $£ 50$, or $£ 100$ ) for the device.

This study will not be used to set prices for self-injection devices, but it will help decision makers understand how important device features are to people. If people do not pay attention to the amounts shown, our analysis will be wrong. We won't get a true measure of the value of self-injection device features to you.

Please help us measure your preferences correctly by carefully considering to the amounts shown before deciding which self-injection device you would choose.

\section{Feature Descriptors}

\section{Description of Self-Injection Devices in the Survey}

All self-injection devices are devices that allow you to inject yourself with medicine at home without having to go to a doctor's office or clinic. When you are ready to begin the injection, you place the end of the device firmly against your skin and start the injection, which can last up to 15 seconds. The needle is hidden in the device, and it is not visible before, during, or after the injection.

Standard devices are disposable pens that are prefilled with medicine and are often stored in the refrigerator. Once the injection is over, you throw away the whole device in a sharps bin.

The hypothetical devices with additional features come with disposable cartridges that are filled with medicine and are often stored in the refrigerator. Once the injection is over, you throw away the cartridge in a sharps bin, but the whole device is reusable for up to 3 years.
Before you can get a device with additional features, your doctor or pharmacist will need to set it up and customize it to your personal needs.

\section{Skin sensor}

Some self-injection devices may have a skin sensor. This feature can detect whether the device is placed on your skin correctly and will not allow the injection to start until the device is in the proper place. If, for any reason, the device loses contact with the skin in the middle of an injection, the sensor will trigger an emergency stop to avoid losing any medicine.

\section{Control of the speed of the injection}

Some self-injection devices may have a speed adjustment feature that allows you to control the speed of the injection.

If a device has this feature, you would be able to increase or decrease the speed at which the medicine is injected into your body. This allows you to inject the medicine at a speed that is most comfortable for you.

In addition, if the device has this feature, you would also be able to pause the injection by pressing a button on the injection device.

\section{Step-by-step on-screen instructions}

Some self-injection devices may have a screen that presents step-by-step instructions while you use the device instead of listing the instructions in a separate booklet or leaflet. These on-screen instructions guide you through every step of the injection, from beginning to end. This feature will guide you throughout the injection process and give you warnings to help you know what to do in case you have a problem with the injection. The feature will also let you know when the injection has been completed.

\section{Injection date reminders}

Some self-injection devices may use information about the time of your last injection to remind you when it is time for your next injection. The reminder will be sent to you by e-mail or text message shortly before you need to take your next injection. This information could also be delivered as an automatic message through an app or through the calendar feature on your smartphone or tablet.

\section{Electronic log}

Some self-injection devices keep a log of the dose and date of each injection so that you and your doctor can monitor your treatment, knowing whether and when you took your medication. This information can be transmitted securely through a wireless connection (using a cellular or Wi-Fi 
network) to a computer, tablet, or smartphone, where you can access and analyze it using a computer program or app. The program or app will also allow you to enter information about your symptoms so that you and your doctor can monitor your health and the severity of your symptoms. If you would like, the program or app will also allow you to share this information with your doctor automatically.

\section{Grip size}

Self-injection devices come in different sizes. Some have grips (the place where you hold the device in your hand) that are designed to make it easier to hold and to inject the medicine. Figure S1 may help you think about the size of different injection devices.

\section{Compensating Variation}

The Compensating Variation (CV) for a given reusable self-injection device compared with the current standard self-injection device with no enhanced features, small grip and no out-of-pocket cost, is defined by the following:

$$
\begin{aligned}
\mathrm{CV}= & -\frac{1}{\beta_{\text {cost }}}\left[\ln \left(\exp \left(\sum_{k=1}^{K}\left[\left(\beta_{k}\right) X_{k 1}\right]\right)\right)\right. \\
& \left.-\ln \left(\exp \left(\sum_{k=1}^{K}\left[\left(\beta_{k}\right) X_{k 0}\right]\right)\right)\right]
\end{aligned}
$$

where $\beta_{\text {cost }}$ is the estimated preference weight for out-ofpocket cost; $\beta_{k}$ represents the preference weights for the $\mathrm{k}$ $\epsilon \mathrm{K}$ attribute, including the alternative specific constant; $X_{0}$ represents the combination of $\mathrm{K}$ attributes (features) in the standard self-injection device; and $X_{1}$ represents the combination of $\mathrm{K}$ attributes (features) in the reusable selfinjection device we want to evaluate. Each log sum term $\left(\ln \left(\exp \left(\sum_{k} \beta_{k}{ }^{\prime} X_{1}\right)\right), \ln \left(\exp \left(\sum_{k} \beta_{k}{ }^{\prime} X_{0}\right)\right)\right)$ is the expected utility from a reusable self-injection device with a specified bundle of enhanced features and the standard selfinjection reference device.

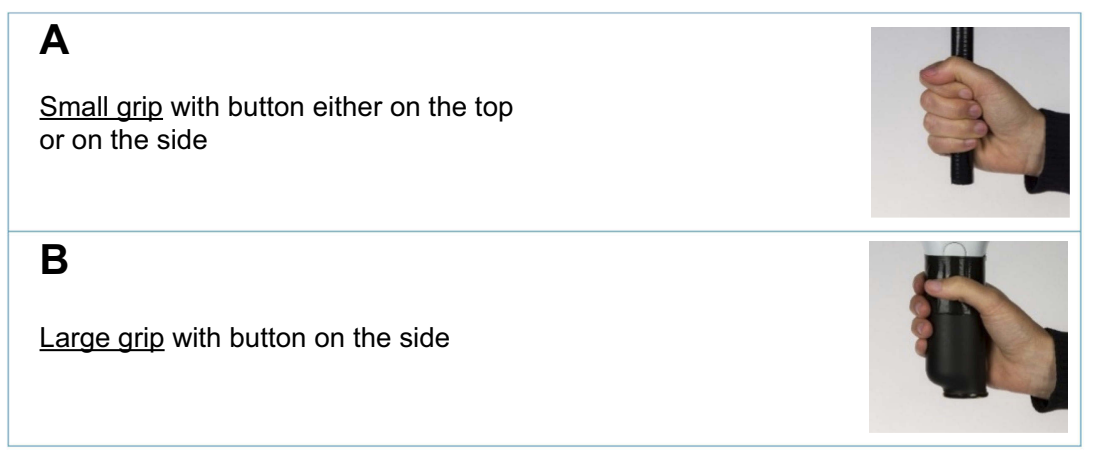

Figure SI Example DCE question. The final experimental design included 40 sets of 3 devices split into 4 blocks of 10 hypothetical choice questions. Patients were randomly assigned to I block of 10 questions. Questions were randomly ordered to avoid ordering effects.

\section{Publish your work in this journal}

Patient Preference and Adherence is an international, peer-reviewed, open access journal that focusing on the growing importance of patient preference and adherence throughout the therapeutic continuum. Patient satisfaction, acceptability, quality of life, compliance, persistence and their role in developing new therapeutic modalities and compounds to optimize clinical outcomes for existing disease states are major areas of interest for the journal. This journal has been accepted for indexing on PubMed Central. The manuscript management system is completely online and includes a very quick and fair peer-review system, which is all easy to use. Visit http:// www.dovepress.com/testimonials.php to read real quotes from published authors. 